\title{
Epidermodysplasia verruciformis and susceptibility to HPV
}

\author{
Tejas Patel $^{\mathrm{a}}$, L. Katie Morrison ${ }^{\mathrm{a}, \mathrm{b}}$, Peter Rady ${ }^{\mathrm{a}, \mathrm{b}}$ and Stephen Tyring ${ }^{\mathrm{a}, \mathrm{b}, *}$ \\ ${ }^{a}$ Center for Clinical Studies, University of Texas Health Science Center, Houston, TX, USA \\ ${ }^{\mathrm{b}}$ Department of Dermatology, University of Texas Health Science Center, Houston, TX, USA
}

\begin{abstract}
Purpose of review: Epidermodysplasia verruciformis has been addressed in depth in the recent literature despite its rarity. The disease is characterized by a persistence in human papillomavirus infections and development of cutaneous malignancies, usually happening more frequently and at a younger age than in the general population. Because of the role of immunodeficiency to viral antigens eventually leading to cancer, EV has become a model for understanding a viral role in cutaneous oncogenesis. Susceptibility loci for EV have been mapped and encoded protein functions are becoming better understood. Discoveries of novel mutations and further study of EV-associated HPV serotypes in lesional and nonlesional skin of affected patients and the general population may help generate a cohesive theory regarding the true role of a defective immune barrier in oncogenesis.
\end{abstract}

Keywords: Cutaneous oncogenesis, epidermodysplasia verruciformis, epidermodysplasia verruciformis-human papillomavirus

\section{Introduction}

Epidermodysplasia verruciformis (EV) was first described in 1922 by Lewandowski and Lutz [1]. Since then it has been studied and cited frequently for its role as a model for viral associated cutaneous oncogenesis. It is characterized by an extreme susceptibility to certain human papillomaviruses [2]. However, these types are not limited to this disorder and can be found in benign and malignant lesions in the normal population [2].

Research has shown genetically preordained deficiencies in cutaneous immunity leave epidermodysplasia verruciformis (EV) patients vulnerable to persistent HPV infection [2]. As explained later, mutations in a family of genes, called EVER genes, code for proteins whose deficiency are responsible for the condition [3]. Since an initial report, evidence for these mutations has been found in EV affected families throughout the

*Corresponding author: Stephen Tyring, MD, Center for Clinical Studies, 6655 Travis, Suite 120, Houston, TX 77030, USA. Tel.: +1 7135288818 ext. 1211; Fax: +1 713528 8848; E-mail: styring@ ccstexas.com. world. The same group has also reported a second susceptibility locus implicating another cause for the disease, and revealing nonallelic heterogeneity in the mechanism [4].

Devising successful treatment options for patients has been challenging and not always fruitful. Many of these therapies have helped induce regression of lesions. However, follow up results have been widely inconsistent, ranging from remission to rapid growth of new lesions upon treatment cessation. As such, there is a continued urgency to find an effective first line treatment and discern more about this disease.

\section{Clinical presentation}

Generally, like all papillomaviridae, HPV infects only the stratified epithelium of the epidermis or mucous membranes. The vast majority of HPV infections are asymptomatic, but there are some which can cause verrucae, and a minority of cases can lead to cancer within the skin and anogenital tract. However, though most warts are recurrent, they are usually few in number compared to the mass of lesions accrued in EV. Addi- 
Table 1

Summary of EVER1 mutations reported in EV

\begin{tabular}{|c|c|c|c|c|}
\hline Mutation & Position (cDNA) & Exon & IVS* & References \\
\hline nonsense & $280(\mathrm{C}$ to $\mathrm{T})$ & 5 & & [3] \\
\hline nonsense & $1726(\mathrm{G}$ to $\mathrm{T})$ & 14 & & [3] \\
\hline nonsense & $744(\mathrm{C}$ to $\mathrm{A})$ & 8 & & [15] \\
\hline splice site (acceptor) & IVS8-2 (A to T) & & 8 & [15] \\
\hline frameshift & insertion CATGT after 916 & 9 & & [16] \\
\hline frameshift & 968 (T deletion) & 9 & & [12] \\
\hline nonsense & $220(\mathrm{C}$ to $\mathrm{T})$ & 4 & & [11] \\
\hline
\end{tabular}

*IVS, intervening sequence, ${ }^{* *}$ heterozygous mutations from the same patients.

tionally, although cutaneous infection with HPV may not cause symptoms in normal patients, EV patients may have lesions with the same types, most specifically the EV-HPV types (including HPV 5, 8, 9, 12, $14,15,17,19,25,36,38,47,50)$ [2]. The greatest concern is centered around HPV types 5, 8, and 14d, which are most commonly associated with malignant transformation of epidermodysplasia verruciformis [5].

On examination, the lesions are polymorphic, including pityriasis versicolor-like macules and flat, wart-like papules that can undergo malignant change [6]. Usually benign forms of EV present with flat, slightly scaly, red-brown macules and pinkish-red plane papules on the face, neck and body. In addition to lesions, during the fourth and fifth decade of life, half of all patients will develop malignancy (primarily Bowen's type carcinoma in situ and invasive squamous cell carcinoma) that occurs mainly on sun exposed area in [6]. As such, malignant forms usually show a higher rate of polymorphic skin lesions due to uncontrolled growth of numerous lesions. These lesions can be more closely described as verruca-like papillomatous lesions or seborrheic keratosis-like lesions. Due to the sheer number of lesions on most EV patients and increased propensity for malignancy, they should be followed closely for development of cancer.

\section{Genetic background}

When trying to obtain inheritance patterns among patient pedigrees, assessment revealed that about $10 \%$ (in a review of 147 case reports) are born to consanguineous parents [7]. One group concluded that because the proportion of affected siblings steadily approached $30 \%$, the mode of EV transmission may likely be autosomal recessive [8]. Reports of X-linked recessive inheritance have also surfaced [9], supporting theories of possible genetic heterogeneity [10]. Regardless of the mode, researchers are still trying to shed light on the molecular basis underlying this disease.
Table 2

Summary of EVER2 mutations reported in EV

\begin{tabular}{lccc}
\hline Mutation* & Position (cDNA) & Exon & References \\
\hline frameshift & 755 (T deletion) & 7 & {$[3]$} \\
nonsense & $1084(\mathrm{G}$ to T) & 9 & {$[3]$} \\
nonsense & $568(\mathrm{C}$ to T) & 6 & {$[14]$} \\
Deletion & $561 \_583$ & 6 & {$[45]$} \\
Nonsense & $188(\mathrm{G}$ to A) & 3 & {$[13]$} \\
\hline
\end{tabular}

*All of the mutations were homozygous and created premature termination codons.

As described earlier, one group has mapped the susceptibililty loci EV1 to chromosome $17 \mathrm{q} 25$ and EV2 to chromosome 2 [3,4]. Since those discoveries, specific mutations in EVER1 and EVER2 within the EV loci have also been reported. All EV patients in two Algerian and two Colombian consanguineous families shared two highly conserved nonsense mutations in the EVER1 and EVER2 genes [3]. After identifying these gene sequences, unique mutations were discovered in EV patients across the world [11-16]. In total, 10 loss of function mutations caused by a variety of mechanisms (nonsense mutation, single nucleotide deletion, splice site mutation, and exon deletion) have been isolated, seven in EVER1 and five in EVER2 (Tables 1 and 2) $[10,17]$.

These two gene sequences code for transmembrane channel-like proteins and belong to the TMC (transmembrane channel-like) gene family; therefore they are also termed TMC6 (EVER1) and TMC8 (EVER2). The available literature indicates that the TMC proteins encoded by EVER genes have features of integral membrane proteins and localize to the endoplasmic reticulum. Recent investigations show the role of zinc-transporting proteins EVER1, EVER2, and ZnT-1, which maintain cellular zinc homeostasis. Apparently, the deregulation of the cellular zinc balance emerges as an important step in the life cycles not only of cutaneous, but also of genital HPVs, although the latter viruses have developed a mechanism by which they can break the barrier and impose a zinc imbalance. This may be a potential model for the mechanism of function 


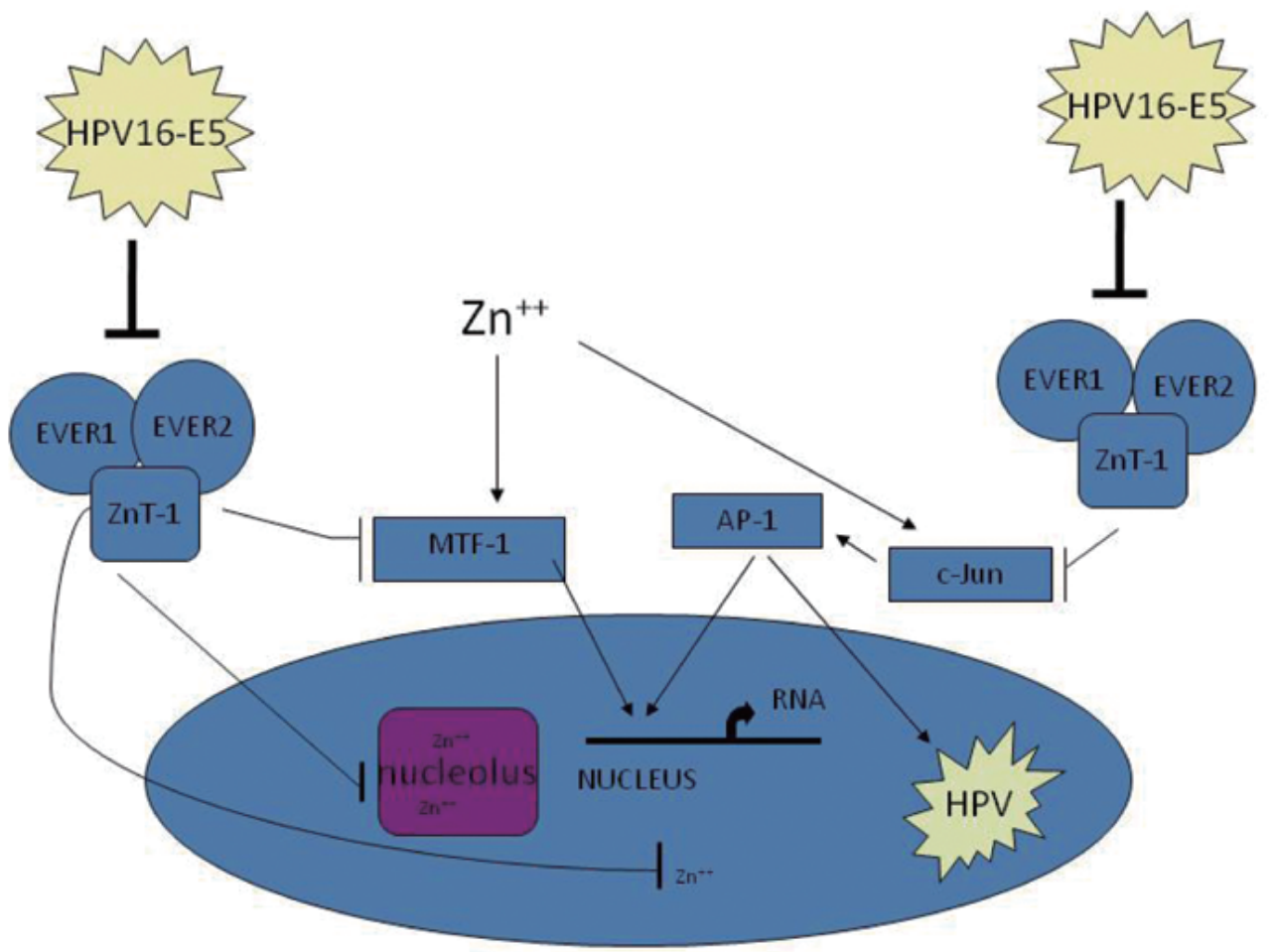

Fig. 1. Role of EVER1 and EVER2 genes in Zn metabolism and protection against pathogenesis of HPVs. EVER1 and EVER 2 proteins form a complex, which binds to the zinc transporter 1 (ZnT-1) and is located in the ER. The ZnT1-EVER complex regulates intracellular zinc distribution by decreasing the nuclear and nucleolar zinc concentration [53]. The decreased nuclear zinc supply interferes with HPV's zinc dependent replication [53]. EVER - ZnT-1 complex also is a negative regulator of metal-responsive transcription factor-1 (MTF-1) and AP-1 transcription (through c-Jun inhibition). MTF-1 transcription factor contributes to various characteristics of cancer biology e.g. angiogenesis, invasion, apoptosis resistance and drug and radiation resistance [54]. AP-1 is an essential transcription factor in the activation of the HPV life cycle [55]. HPV16 E5 protein binds to the EVER - ZnT-1 complex and inhibits its functions [53].

of the anti-HPV barrier [18]. Along with this, Orth G observed EV-specific HPVs (betapapillomaviruses) are defective for an important growth-promoting function encoded by an E5/E8 gene present in other HPVs, and inactivation of EVER proteins may compensate for the missing viral function [19] (Figs 1 and 2 for mechanism of molecular pathogenesis).

Despite all the above findings, a significant portion (24.4\%) of EV patients cannot be explained by the EVER1 or EVER2 mutations [10]. One group reported a Turkish family with unexplained inheritance patterns [20]. An autosomal dominant inheritance lacking EVER1 or EVER2 mutation was shown recently in a father/son pair [21]. Zavattaro et al. described the identification of defective Fas function and variation of the perforin gene in an epidermodysplasia verruciformis patient lacking EVER1 and EVER2 mutations [22]. It is possible yet undiscovered genetic loci or genes within the EV2 locus can be implicated. These results show further chromosomal studies on this unique group of patients are necessary.

\section{Defective immunity in EV pathogenesis:}

Alternative hypotheses revolve around epidermodysplasia verruciformis representing a primary deficiency of intrinsic immunity, and that these EVER proteins function as restriction factors for EV specific HPVs in keratinocytes [9]. As an example, in a case report, a patient with clinically and virologically typical epidermodysplasia verruciformis had a lack of EVER mutations and CD8+ T-cell lymphocytopenia [23]. This lends support to a potential defective immunity in control of cutaneous HPV infection.

Interestingly, unaffected patients who have no previous evidence of epidermodysplasia verruciformis can go on to develop a similar syndrome when immunodeficient. Recently, a group described two cases of EV-like syndrome in HIV-positive patients. Due to impaired cell-mediated immunity, the term "acquired epidermodysplasia verruciformis" was used to describe EV developing in the immunocompromised host [24]. 


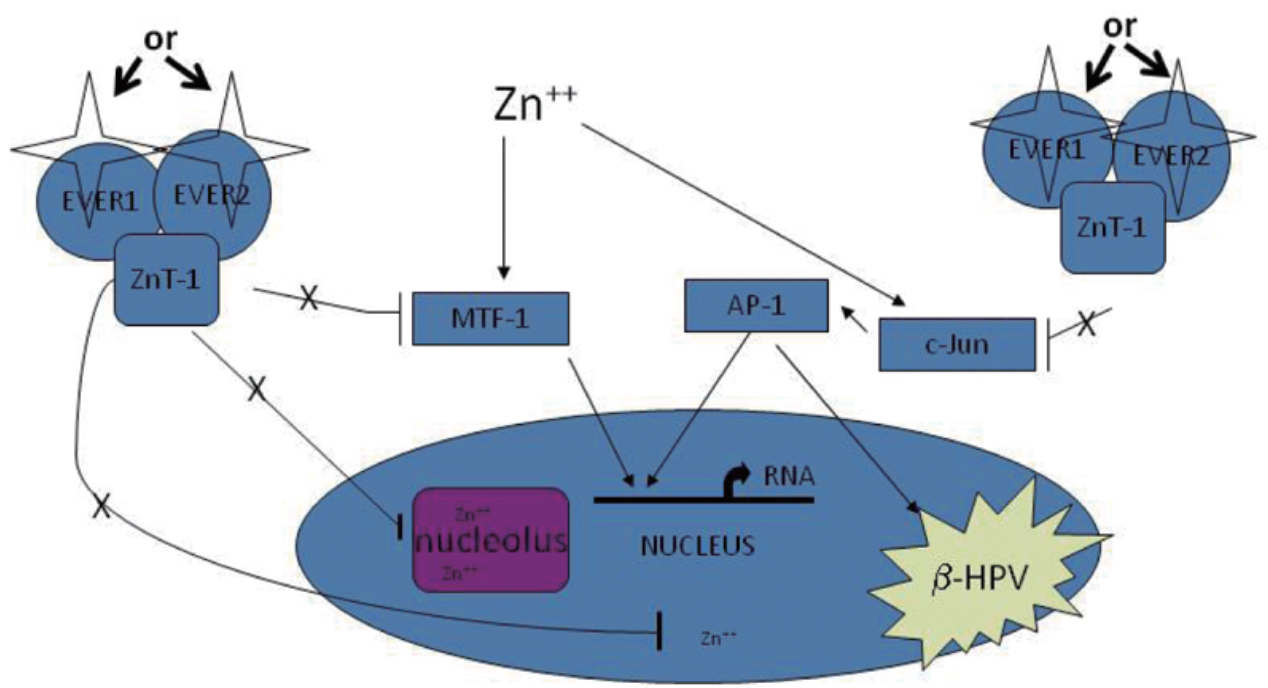

Fig. 2. Effect of mutations in EVER1 or EVER2 genes on Zn metabolism and on host restriction against EV-HPVs in EV patients. The EV-associated HPVs do not have a functional E5 protein encoded in the viral genome, which can be a clue for host restriction of these virus types. Approximately $75 \%$ of patients with inherited EV have mutations in one of the EVER genes [10]. A mutation in one of the EVER genes is sufficient for disease onset. These truncating mutations $(\neg)$ lead to the lack of the proper EVER-ZnT-1 complex formation, and the host restriction mechanisms against the EV-HPVs are turned off (X) [53].

Another case report supported the hypothesis that the combination of immunodeficiency and a susceptibility allele may contribute to the differences in occurrence of EV in HIV-positive patients [25]. This has also been shown to happen in association with common variable immunodeficiency (CVID) syndrome, where epidermodysplasia verruciformis manifested as flat warts and squamous cell carcinomas in a 21-year-old Hispanic woman. Human papillomavirus (HPV) typing by polymerase chain reaction using an EV-HPV primer system, cloning, and sequencing detected HPV-8 and HPV-23 DNAs in a biopsy sample, in the second reported case with CVID [26].

Studying related immunodeficiency disorders which display particular susceptibility to HPV infection may reveal some possible links to the pathophysiology of this genodermatosis. WILD syndrome (warts, immunodeficiency, lymphedema, dysplasia) presents with similar skin lesions to EV along with primary lymphedema, anogenital dysplasias, and depressed cellmediated immunity. Distinctively, the skin lesions are consistently infected with alpha HPV types, as opposed to the beta types in EV [27]. The warts in WHIM syndrome are accompanied by hypogammaglobulinemia, infection, and myelokathexis. Here, a gene mutant located on 2 q21 causes a chemokine receptor protein to remain in a constant activated state [28]. Even broader combined immunodeficiency seen in DOCK8 mutations can lead to recurrent sinopulmonary and cuta- neous viral infections, leading to multiple verruca from cutaneous HPV infection [29]. These examples give insight into the possibility of a deficiency in primary immunity leading to epidermodysplasia verruciformis. (See reviews by Bachelerie, Freeman, and Zhang in this issue for further discussion of WHIM syndrome and DOCK8 deficiency.)

\section{Role in cutaneous oncogenesis}

In the general population, surveillance for genital tract cancers in patients with human papillomavirus infection is a generally accepted part of preventative medicine. This is based on the proven causative relationship between HPV infection and squamous cell carcinoma of the genital tract. Development of the quadrivalent vaccine against HPV 6, 11, 16, and 18 as well as the bivalent vaccine against HPV 16 and 18 are aimed at decreasing future incidence of cervical cancer [10]. Although this is the status quo for genital lesions, the relationship of HPV to cutaneous oncogenesis is not as well defined.

The role of ultraviolet radiation as a causative agent in the development of nonmelanoma skin cancer (NM$\mathrm{SC}$ ) is well established. Research has shown that UV exposure can lead to mutations in DNA, increasing the risk of cancer [20]. EV associated HPV types have been isolated in active lesions; the vast majority (90\%) 
of EV cancers have been shown to contain copies of HPV 5 and 8 . One study examined seroreactivity to six EV HPV types, five of which had increased seroreactivity in squamous cell carcinoma cases (odds ratio of 14.7 for HPV 8). The results indicate that EVHPV serorecognition is nonspecifically associated with nonmelanoma skin cancer and suggest that EV-HPVdirected seroresponses are induced upon skin cancer formation [30]. A case control study examined the link between EVER2 variation and HPV seropositivity, along with squamous cell cancer risk. The data showed variant genotypes were associated with beta HPV seropositivity with an odds ratio of 2.3 , specifically with HPV 5 and $8(\mathrm{OR}=2.4)$ and multiple HPV types $(\mathrm{OR}=2.7)$. Furthermore, variant genotype was also related to squamous cell carcinoma risk [adjusted OR for homozygous variant versus homozygous wild type for the EVER2 polymorphism 1.7]. This provides evidence for a role of genetic variation in the EVER2 gene in beta-HPV infection and risk of SCC, shedding light on the link between HPVs and skin cancers [31].

Though it is logical to suspect HPV in developing cutaneous malignancies, the association does not necessarily confirm a casual relationship [32]. Furthermore, PCR detection of HPV DNA has revealed its existence in normal skin of immunocompromised and immunocompetent patients alike. As such, the likelihood of a direct oncogenic effect is decreased [33]. One study even found that HPV DNA is common in superficial layers of lesions, but is not necessarily present throughout tumors [34]. After stripping the upper epidermis of malignant skin lesions, HPV DNA detection dropped 3.2 to 7.9 fold. This opened the possibility that that HPV positivity in tumor biopsies by PCR may merely reflect contamination of the lesion surface and are not related to tumor below [35].

While the relationship between HPV and cutaneous malignancy may not be so clear cut, there are still possibilities of HPV playing a more indirect role. One view is described by the findings of Pfister et al., in which there was no difference between the low- and high-grade actinic keratoses either in terms of EV-HPV DNA prevalence $(85 \%)$ or the results of serological study using HPV8 virus-like particles. The detection rate of EV-HPVs was lower in skin cancers $(45 \%)$ and Bowen's disease $(33 \%)$. This would suggest involvement of EV-HPVs in the early stages of cutaneous oncogenesis [36]. Another study suggests that persistence of HPV is not necessary to maintian of the malignant phenotype of individual NMSC cells. Although a passenger state cannot be excluded, the data are compatible with a carcinogenic role of HPV in early steps of tumor development [37]. Another theory suggests HPV infection acts as a co-carcinogen to ultraviolet radiation. In the general population, actinic keratosis and nonmelanoma skin cancer develop on sun exposed areas; however these lesions occur with greater frequency and at an earlier age in epidermodysplasia verruciformis patients. Nevertheless, more research may be needed as indicated by an increased risk for HPV infection in patients with sunburn history compared to a lower risk for EV HPV DNA detection in patients with an overall greater lifetime sun exposure in nonerythematogenic doses $[17,38]$.

\section{Treatment}

Finding ways to address skin lesions in epidermodysplasia verruciformis patients is a constant struggle. Because of the above described mechanisms, patients need to be closely surveyed for malignant transformation of these lesions. Once malignancies are detected, treatment is mainly designed based on the extent of disease. Localized malignancies can be removed surgically, but the extensive, nonmalignant lesions need a more practical therapy. For years, retinoids have been touted for their endogenous antiproliferative effect through control of epithelial cell differentiation [39]. As such, they would be logical agents to counteract HPV infection with associated epithelial hyperplasia. Furthermore, they may also aid in reducing progression of EV lesions to dysplasia and oncogenicity by maintaining normal epithelial differentiation [40]. In a case report involving a 25 year old woman, $0.5-1 \mathrm{mg} / \mathrm{kg} /$ day acitretin for 6 months improved skin lesions slightly. However, upon discontinuation of treatment, the lesions returned, and the patient declined further treatment [40].

Another avenue explored that has provided more effective results was acitretin combined with interferon therapy. Interferons are known for their antiviral activity and can also promote cellular differentiation, thereby inhibiting malignant cell proliferation [39]. Anadolu et al. presented a case of EV that was treated with a combination of acitretin and interferon alfa- $2 \mathrm{a}$ for three months with marked improvement. Though the lesions recurred after discontinuating treatment, the patient restarted combination therapy for 4 months, followed by acitretin alone for 3 months. Upon follow up a year after the end of the second course, the patient was lesion-free on her face with few residual flat warts on her hands [39]. In another case report, pegylated in- 
terferon alfa- $2 b$ and acitretin was given to a 43 year old female with multiple squamous cell carcinomas in the oral and genital mucosa and widespread verrucous lesions. The patient saw a marked reduction of flat warts and no recurrence of cancer during treatment [41].

Side effects of lip dryness, dry skin, and burning sensation are common in retinoid therapy. Therefore, EV patients may need a viable alternative. In one case, Hayashi et al. treated a patient with intolerable retinoid side effects with topical tacalcitol, a vitamin D analog. Not only did the largest lesion regress within six months of therapy, but it also prevented new carcinomas over the following three years [42].

Other therapies include cimetidine, a histamine type 2 receptor antagonist, which has demonstrated immunomodulating properties. It has been used to treat planar, plantar, and common warts in patients not responding to other therapies. One case report of a patient who started treatment with oral cimetidine (40 $\mathrm{mg} / \mathrm{kg}$ per day) demonstrated a marked improvement after 3 months of therapy, with no relapse at a 6-month follow-up [43]. However, de Oliveira et al. used the same treatment regimen in eight EV patients with unsatisfactory results [44].

Imiquimod is a topical immune modulator that is usually used for common and genital warts, as well as squamous cell carcinoma and Bowen's disease. Berthelot et al. described a patient with EV and a novel homozygous gene mutation of an EVER2 gene who was treated successfully with topical imiquimod application for 5 days/week for 3 months [45]. This topical therapeutic was also used along with systemic interferon therapy in an EV patient with Bowen's disease and actinic keratoses [46].

In a novel therapy, Karrer et al. performed photodynamic therapy (PDT) using a 20\% 5-aminolaevulinic acid ointment applied for $6 \mathrm{~h}$ to the lesions and irradiating using an incoherent light source (lambda $=580$ $740 \mathrm{~nm}, 160 \mathrm{~mW} / \mathrm{cm} 2,160 \mathrm{~J} / \mathrm{cm} 2$ ). Following PDT, blistering and crusting of the lesions occurred, but these healed completely within 2-3 weeks without scarring, and the cosmetic result was excellent. Six months after PDT a skin biopsy was taken. In situ hybridization was positive for HPV type 8 in skin, which was clinically and histologically normal. Twelve months after PDT, a few lesions had recurred on the hands. The authors conceded that though permanent cure of EV cannot be achieved by any therapy at present and single lesions continued to appear in this patient, annual PDT might result in better control of HPV-induced lesions [47].

Though traditional therapies such as cidofovir may be effective against other papillomavirus associated conditions, it has been unsuccessful in treating an EV patient with multiple cutaneous lesions [48].

Treatment in epidermodysplasia verruciformis patients with concurrent HIV infection requires specialized attention as several studies have shown most treatments to be ineffective. Topical imiquimod in two HIV positive half brothers did not show improvement [49]. Davison et al. tried imiquimod, 5-fluorouracil, and isotretinoin, all without success $[17,50]$. The effects of HAART therapy in the improvement of EV disease states have not been clearly defined. One group found it to have no effect [51], while Haas et al. reported a patient being treated with highly active antiretroviral therapy who experienced improvement of EV lesions [52].

\section{Conclusion}

Epidermodysplasia verruciformis is a rare genetic disorder, which is manifest by cutaneous immunodeficiency and susceptibility to repeated and persistent HPV infection. This is the mechanism that leads to an increased propensity for skin malignancies. Advances, mainly in the last decade, have elucidated the genetic basis for the disease with the discovery of susceptibility loci and related mutations. Further research is still needed in this area to define the inheritance pattern and what role EV HPV DNA plays in cutaneous oncogenesis. It has been implicated to be related at several different levels of skin cancer growth. Treatment for this disease can be effective; however, there seems to be much variation within patient populations and also a lack of documentation. Though there are a plethora of options, none seem to be curative, and lesions usually recur after treatment cessation.

\section{References}

[1] F. Lewandowsky and L.W., A case of a previously undescribed skin disease (epidermodysplasia verruciformis) [in German], Arch Dermatol Syphilol (141), 193-203.

[2] J.E. Lane, P.H. Bowman and D.J. Cohen, Epidermodysplasia verruciformis, South Med J 96(6) (2003), 613-615.

[3] N. Ramoz et al., Mutations in two adjacent novel genes are associated with epidermodysplasia verruciformis, Nat Genet 32(4) (2002), 579-581.

[4] N. Ramoz et al., Evidence for a nonallelic heterogeneity of epidermodysplasia verruciformis with two susceptibility loci mapped to chromosome regions 2p21-p24 and 17q25, J Invest Dermatol 114(6) (2000), 1148-1153.

[5] W.R. Oliveira et al., Skin cancer in epidermodysplasia verruciformis patients from Brazil, J Eur Acad Dermatol Venereol 20(9) (2006), 1154-1156. 
[6] W.R. de Oliveira et al., Clinical aspects of epidermodysplasia verruciformis, J Eur Acad Dermatol Venereol 17(4) (2003), 394-398.

[7] M.A. Lutzner, Epidermodysplasia verruciformis. An autosomal recessive disease characterized by viral warts and skin cancer. A model for viral oncogenesis, Bull Cancer $\mathbf{6 5}(2)$ (1978), 169-182.

[8] V.N. Sehgal, A. Luthra and P. Bajaj, Epidermodysplasia verruciformis: 14 members of a pedigree with an intriguing squamous cell carcinoma transformation, Int J Dermatol 41(8) (2002), 500-503

[9] E.J. Androphy, I. Dvoretzky and D.R. Lowy, X-linked inheritance of epidermodysplasia verruciformis. Genetic and virologic studies of a kindred, Arch Dermatol 121(7) (1985), 864-868.

[10] G. Orth, Genetics of epidermodysplasia verruciformis: Insights into host defense against papillomaviruses, Semin Immunol 18(6) (2006), 362-374.

[11] S. Aochi et al., A novel homozygous mutation of the EVER1/TMC6 gene in a Japanese patient with epidermodysplasia verruciformis, Br J Dermatol 157(6) (2007), 1265-1266.

[12] M.D. Gober et al., Novel homozygous frameshift mutation of EVER1 gene in an epidermodysplasia verruciformis patient, J Invest Dermatol 127(4) (2007), 817-820.

[13] P.L. Rady et al., Novel homozygous nonsense TMC8 mutation detected in patients with epidermodysplasia verruciformis from a Brazilian family, Br J Dermatol 157(4) (2007), 831833.

[14] X.K. Sun, J.F. Chen and A.E. Xu, A homozygous nonsense mutation in the EVER2 gene leads to epidermodysplasia verruciformis, Clin Exp Dermatol 30(5) (2005), 573-574.

[15] G. Tate et al., Novel mutations of EVER1/TMC6 gene in a Japanese patient with epidermodysplasia verruciformis, $J$ Hum Genet 49(4) (2004), 223-225.

[16] Y.G. Zuo et al., Identification of a novel mutation and a genetic polymorphism of EVER1 gene in two families with epidermodysplasia verruciformis, J Dermatol Sci 44(3) (2006), $153-159$.

[17] A. Gewirtzman, B. Bartlett and S. Tyring, Epidermodysplasia verruciformis and human papilloma virus, Curr Opin Infect Dis 21(2) (2008), 141-146.

[18] M. Lazarczyk et al., The EVER proteins as a natural barrier against papillomaviruses: a new insight into the pathogenesis of human papillomavirus infections, Microbiol Mol Biol Rev 73(2) (2009), 348-370.

[19] G. Orth, Host defenses against human papillomaviruses: lessons from epidermodysplasia verruciformis, Curr Top Microbiol Immunol 321 (2008), 59-83.

[20] B. Akgul et al., A distinct variant of Epidermodysplasia verruciformis in a Turkish family lacking EVER1 and EVER2 mutations, J Dermatol Sci 46(3) (2007), 214-216.

[21] D.F. McDermott et al., Autosomal dominant epidermodysplasia verruciformis lacking a known EVER1 or EVER2 mutation, Pediatr Dermatol 26(3) (2009), 306-310.

[22] E. Zavattaro et al., Identification of defective Fas function and variation of the perforin gene in an epidermodysplasia verruciformis patient lacking EVER1 and EVER2 mutations, J Invest Dermatol 128(3) (2008), 732-735.

[23] B. Azzimonti et al., CD8+ T-cell lymphocytopenia and lack of EVER mutations in a patient with clinically and virologically typical epidermodysplasia verruciformis, Arch Dermatol 141(10) (2005), 1323-1325.

[24] H.D. Rogers et al., Acquired epidermodysplasia verruciformis, J Am Acad Dermatol 60(2) (2009), 315-320.
[25] E. Hohenstein et al., Epidermodysplasia verruciformis in a HIV-positive patient homozygous for the c917A->T polymorphism in the TMC8/EVER2 gene, Dermatology 218(2) (2009), 114-118.

[26] J. Vu et al., Common variable immunodeficiency syndrome associated with epidermodysplasia verruciformis, Am J Clin Dermatol 8(5) (2007), 307-310.

[27] A. Kreuter et al., A human papillomavirus-associated disease with disseminated warts, depressed cell-mediated immunity, primary lymphedema, and anogenital dysplasia: WILD syndrome, Arch Dermatol 144(3) (2008), 366-372.

[28] P.J. McCormick et al., Impaired recruitment of Grk6 and betaArrestin 2 causes delayed internalization and desensitization of a WHIM syndrome-associated CXCR4 mutant receptor, PLoS One 4(12) (2009), e8102.

[29] Q. Zhang et al., Combined immunodeficiency associated with DOCK8 mutations, N Engl J Med 361(21) (2009), 2046-2055.

[30] M.C. Feltkamp et al., Seroreactivity to epidermodysplasia verruciformis-related human papillomavirus types is associated with nonmelanoma skin cancer, Cancer Res 63(10) (2003), 2695-2700.

[31] A.S. Patel et al., Cutaneous human papillomavirus infection, the EVER2 gene and incidence of squamous cell carcinoma: a case-control study, Int J Cancer 122(10) (2008), 2377-2379.

[32] S. Majewski and S. Jablonska, Do epidermodysplasia verruciformis human papillomaviruses contribute to malignant and benign epidermal proliferations? Arch Dermatol 138(5) (2002), 649-654.

[33] J.C. Sterling, Human papillomaviruses and skin cancer, J Clin Virol 32(Suppl 1) (2005), S67-S71.

[34] O. Forslund et al., High prevalence of cutaneous human papillomavirus DNA on the top of skin tumors but not in "Stripped" biopsies from the same tumors, J Invest Dermatol 123(2) (2004), 388-394.

[35] S. Majewski and S. Jablonska, Current views on the role of human papillomaviruses in cutaneous oncogenesis, Int J Dermatol 45(3) (2006), 192-196.

[36] H. Pfister et al., High prevalence of epidermodysplasia verruciformis-associated human papillomavirus DNA in actinic keratoses of the immunocompetent population, Arch Dermatol Res 295(7) (2003), 273-279.

[37] S.J. Weissenborn et al., Human papillomavirus-DNA loads in actinic keratoses exceed those in non-melanoma skin cancers, J Invest Dermatol 125(1) (2005), 93-97.

[38] F. Termorshuizen et al., Sunlight exposure and (sero) prevalence of epidermodysplasia verruciformis-associated human papillomavirus, J Invest Dermatol 122(6) (2004), 1456-1462.

[39] R. Anadolu et al., Treatment of epidermodysplasia verruciformis with a combination of acitretin and interferon alfa-2a, J Am Acad Dermatol 45(2) (2001), 296-299.

[40] F. Iraji and G. Faghihi, Epidermodysplasia verruciformis: association with isolated IgM deficiency and response to treatment with acitretin, Clin Exp Dermatol 25(1) (2000), 41-43.

[41] E. Gubinelli et al., Epidermodysplasia verruciformis with multiple mucosal carcinomas treated with pegylated interferon alfa and acitretin, J Dermatolog Treat 14(3) (2003), 184-188.

[42] J. Hayashi et al., Treatment of localized epidermodysplasia verruciformis with tacalcitol ointment, Int J Dermatol 41(11) (2002), 817-820.

[43] G. Micali et al., Cimetidine therapy for epidermodysplasia verruciformis, J Am Acad Dermatol 48(2 Suppl) (2003), S9S10.

[44] W.R. de Oliveira, C.F. Neto and E.A. Rivitti, The lack of a clinical effect of cimetidine in the treatment of epidermodys- 
plasia verruciformis, J Am Acad Dermatol 50(6) (2004), e14; author reply e15.

[45] C. Berthelot et al., Treatment of a patient with epidermodysplasia verruciformis carrying a novel EVER2 mutation with imiquimod, J Am Acad Dermatol 56(5) (2007), 882-886.

[46] E.B. Baskan et al., A case of epidermodysplasia verruciformis associated with squamous cell carcinoma and Bowen's disease: a therapeutic challenge, J Dermatolog Treat 17(3) (2006), 179-183

[47] S. Karrer et al., Epidermodysplasia verruciformis treated using topical 5-aminolaevulinic acid photodynamic therapy, $\mathrm{Br} \mathrm{J}$ Dermatol 140(5) (1999), 935-938.

[48] W. Preiser et al., No apparent effect of cidofovir in epidermodysplasia verruciformis, J Clin Virol 16(1) (2000), 55-57.

[49] W. Hu et al., Epidermodysplasia verruciformis in two half brothers with HIV infection, J Cutan Med Surg 8(5) (2004), 357-360.

[50] S.C. Davison et al., Epidermodysplasia verruciformis-like eruption associated with HIV infection, Clin Exp Dermatol 29(3) (2004), 311-312.
[51] D. Carre et al., Epidermodysplasia verruciformis in a patient with HIV infection: no response to highly active antiretroviral therapy, Int J Dermatol 42(4) (2003), 296-300.

[52] N. Haas et al., Remission of epidermodysplasia verruciformislike skin eruption after highly active antiretroviral therapy in a human immunodeficiency virus-positive patient, $\mathrm{Br} J \mathrm{Der}$ matol 145(4) (2001), 669-670.

[53] M. Lazarczyk et al., Regulation of cellular zinc balance as a potential mechanism of EVER-mediated protection against pathogenesis by cutaneous oncogenic human papillomaviruses, J Exp Med 205(1) (2008), 35-42.

[54] B.J. Murphy, Regulation of malignant progression by the hypoxia-sensitive transcription factors HIF-1alpha and MTF1, Comp Biochem Physiol B Biochem Mol Biol 139(3) (2004), 495-507.

[55] P.M. Howley and D.R.L., Papillomaviruses and their replication, Fields Virology 2 (2001), Philadelphia: Lippincott Williams and Wilkins, 2197-2230. 


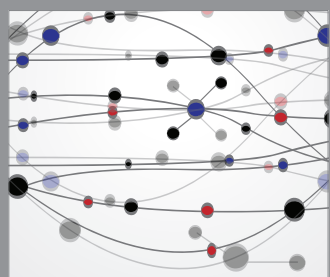

The Scientific World Journal
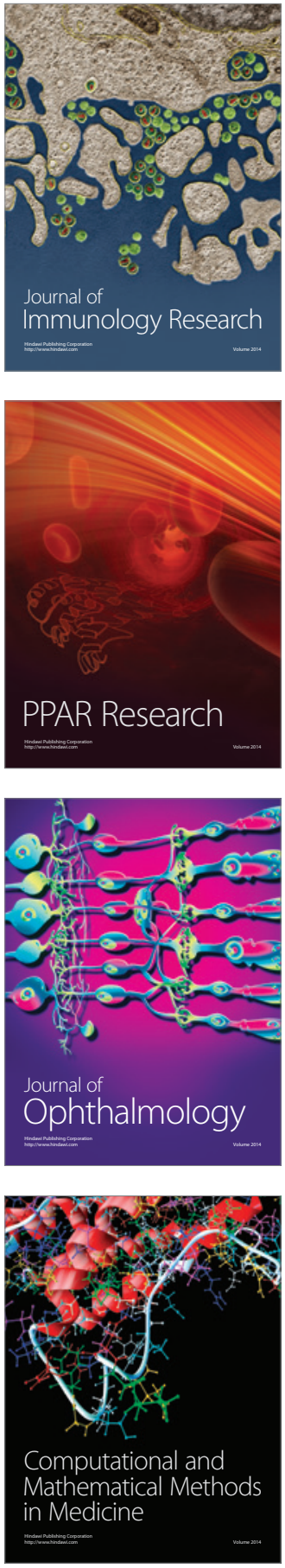

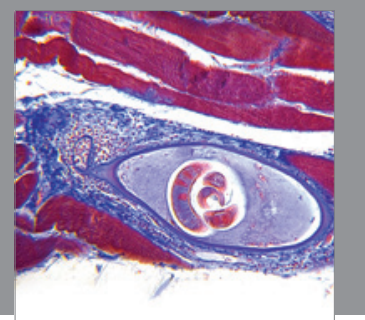

Gastroenterology

Research and Practice
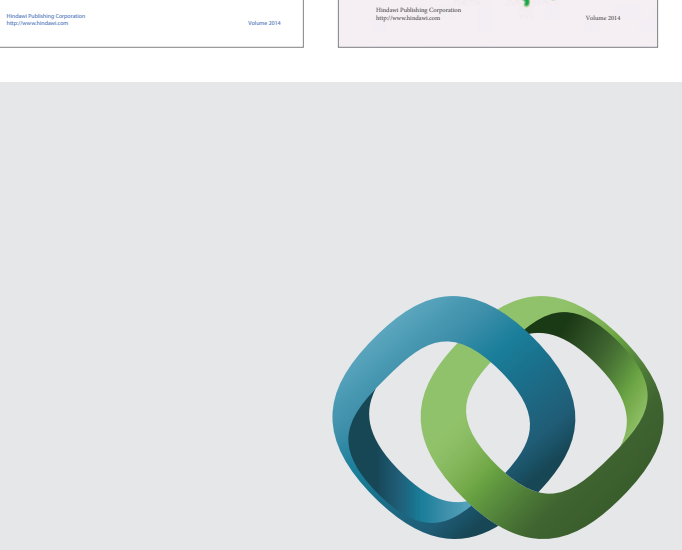

\section{Hindawi}

Submit your manuscripts at

http://www.hindawi.com
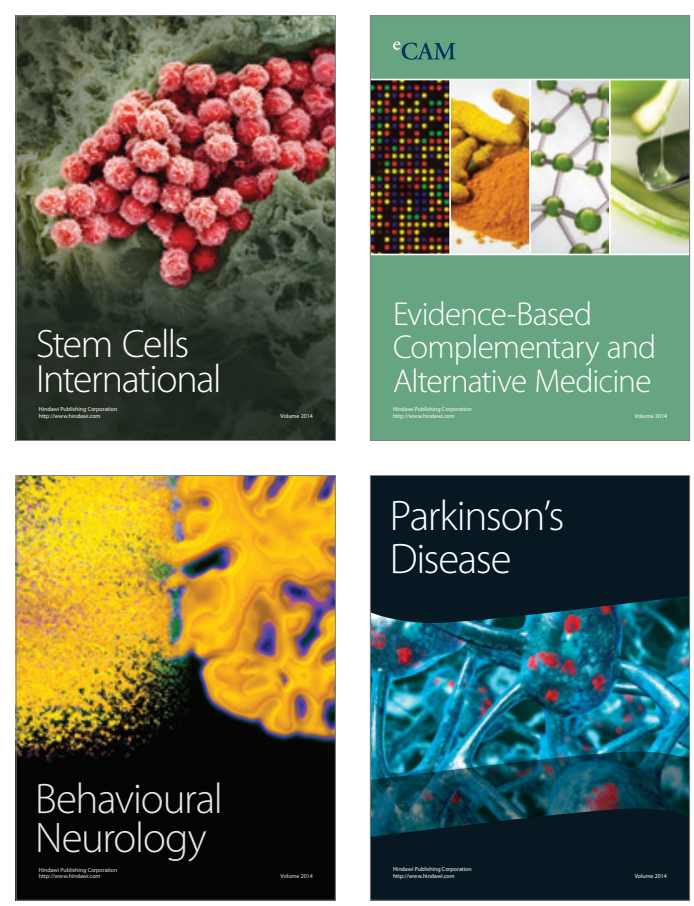

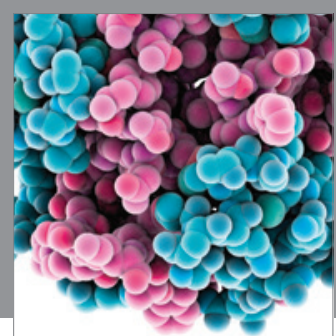

Journal of
Diabetes Research

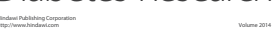

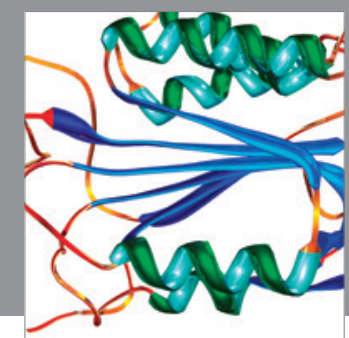

Disease Markers
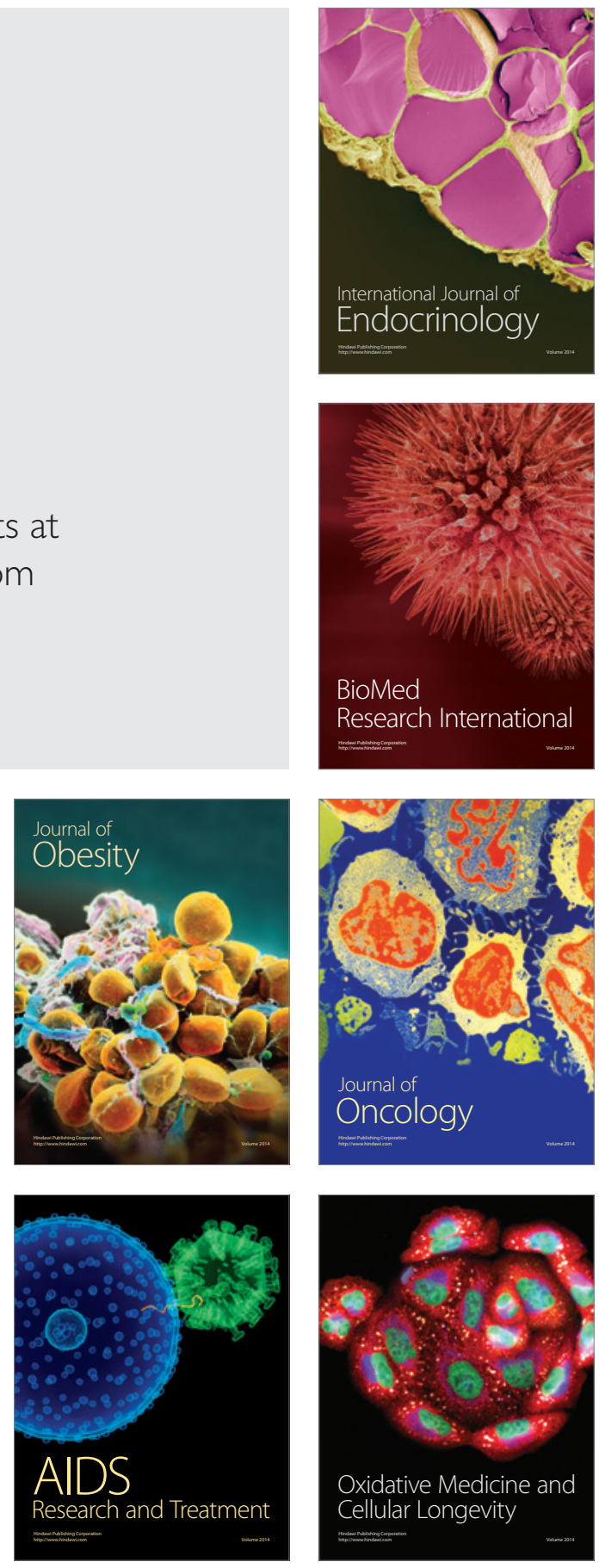